\title{
Static voltage stability analysis using generalized regression neural network
}

\begin{abstract}
The ability of power system to maintain steady voltage at all the buses after happening a disturbance from a given initial operation condition is defined the voltage stability in the system. The focus of this paper is on voltage stability monitoring using the generalized regression neural network by improving algorithm. In this paper, to identify load buses and certain operation condition, the static voltage stability method in power systems is presented. Based on load buses, the index of voltage stability is obtained from the voltage equation derived from a two bus network. The proposed methods are tested on the IEEE-14 bus test system.
\end{abstract}

Keyword: Voltage stability; Generalized regression neural network; Thevenin equivalent circuit; K-fold cross-validation method 